\title{
A method for the fast estimation of a battery entropy-variation high-resolution curve - Application on a commercial $\mathrm{LiFePO}_{4} /$ graphite cell
}

\author{
Nicolas Damay ${ }^{\mathrm{a}, \mathrm{b}}$, Christophe Forgez ${ }^{\mathrm{a}, *}$, Marie-Pierre Bichat $^{\mathrm{b}}$, Guy Friedrich ${ }^{\mathrm{a}}$ \\ ${ }^{a}$ Sorbonne universités, Université de technologie de Compiègne \\ Laboratoire d'Electromécanique de Compiègne, EA 1006 \\ Centre de recherche Royallieu, CS 60319, 60203 Compiègne cedex (France) \\ ${ }^{b} E_{4} V, 9$ avenue Georges Auric, 72000 Le Mans (France)
}

\begin{abstract}
The entropy-variation of a battery is responsible for heat generation or consumption during operation and its prior measurement is mandatory for developing a thermal model. It is generally done through the potentiometric method which is considered as a reference. However, it requires several days or weeks to get a look-up table with a 5 or $10 \%$ SoC (State of Charge) resolution. In this study, a calorimetric method based on the inversion of a thermal model is proposed for the fast estimation of a nearly continuous curve of entropy-variation. This is achieved by separating the heats produced while charging and discharging the battery. The entropy-variation is then deduced from the extracted entropic heat. The proposed method is validated by comparing the results obtained with several current rates to measurements made with the potentiometric method.
\end{abstract}

Keywords: Batteries, Thermal Model Inversion, Heat Generation, Entropy Variation.

\section{Introduction}

Increasing lifetime of batteries is an important issue to make electric vehicles more attractive [1]. It can be achieved through better electrode materials or by slowing down aging mechanisms. More specifically, the main aging factor during operation is considered to be the SEI (Solid Electrolyte Interphase) growth $[2,3]$. Because it is accelerated within a hot battery $[4,5]$, mastering the temperature during function is paramount [6]. Hence, the battery and its cooling system have to be sized optimally and a thermal model is very helpful to achieve this goal $[7,8,9]$.

The thermal modeling of batteries has been widely studied, using methods such as finite element methods $[10,11,12]$, equivalent electric circuit $[13,14,15]$ or solving partial differential equations [16, 17]. Though these approaches are different, they have a common issue in modeling the heat sources [18]. Electrical losses, not studied here, are highly non linear since they depend on the current, the temperature, the state of charge (SoC) and the state of health $(\mathrm{SoH})[19,20,21]$. On the contrary, the entropic heat is easy to model, but the prior measurement of the entropy variation via the classical potentiometric method (presented thereafter) requires very long tests that are hardly compatible with an industrial context $[22,23,24]$.

\footnotetext{
* Corresponding author Forgez)
}

A calorimetric method is presented in this article for the fast determination of the entropy-variation with a high resolution [25]. A simple thermal model is used to estimate the heat generation of a battery [16], from which the entropic heat is deduced. The next part is dedicated to the theoretical background of the proposed method. The thermal model used for estimating the heat generation is presented in a third part, along with its parameter determination. Finally, the entropy variation of a commercial $\mathrm{LiFePO}_{4} /$ graphite cell is determined thanks to the proposed method. These results are compared with those obtained thanks to the potentiometric method, which is considered as the reference.

\section{Theoretical background}

The entropy variation $\Delta S$ is related to the structural changes of the active materials during operation. The corresponding energy variations may result in heat generation or consumption depending on the SoC and the current sign. This behavior can be modeled by the Equation (1) where $I$ is the current, $T$ is the absolute temperature (in Kelvin), $n$ is the number of exchanged electrons during the reaction ( $n=1$ here) and $F$ is the Faraday constant [18].

$$
\dot{Q}_{\Delta S}=I T \frac{\Delta S(S o C)}{n F}=I T \frac{\partial U_{o c}}{\partial T}(S o C)
$$

The term $\Delta S / n F$ can be replaced by the variation of the open-circuit voltage $(\mathrm{OCV})$ regarding temperature 
$\partial U_{o c} / \partial T$ [18]. For more convenience, the latter will also be called "entropy variation" in this paper.

\subsection{Potentiometric method}

In the frame of the potentiometric method, the cell to be characterized is put into a climatic chamber, prepared at a given $\mathrm{SoC}$ and allowed to rest until its OCV is stabilized. Then, a thermal cycle is imposed and the corresponding OCV variation is measured to calculate $\partial U_{o c} / \partial T$ $[22]$.

The process is repeated at other SoC in order to obtain a lookup table covering the whole operation range. Each measurement at a given SoC requires several hours (about half a day) because of the very long time needed for the cell to reach its equilibrium after a temperature change.

\subsection{Proposed calorimetric method}

A calorimetric method is based on the cell heat-generation study. According to Forgez et al. [8], there are four heat sources in a battery: electrical losses, entropic heat, heat generated by side reaction(s) and heat of mixing. Concerning the studied battery, side reactions are mostly aging reactions that are slow enough for their heat generation to be neglected. The heat of mixing is negative during the creation of concentration gradients and is positive when these gradients disappear (the sum being zero) [18]. Its contribution is maximal after current changes and is minor compared to electrical losses [16]. As a consequence, the heat of mixing is neglected in this method. As a result, only electrical losses and entropic heat have been considered in the total heat generation $\dot{Q}$ (Equation (2)).

$$
\dot{Q}=I \times \Delta U(I, T, S o C)+I T \frac{\partial U_{o c}}{\partial T}(S o C)
$$

$\Delta U$ is the "overvoltage" and it depends on the current, the temperature and the SoC. It is due to several phenomena like voltage drop in the electrolyte, charge transfer and diffusion within the active materials. The latter is the slowest one and, within a $\mathrm{LiFePO}_{4} /$ graphite cell, it is similar to a limited diffusion that reaches a quasi-steady state after a few minutes [21]. Consequently, it can be approximated by an equivalent electrical resistance $R$ which is defined as the overvoltage divided by the current. It is written $R_{C h}$ for the charge and $R_{D c h}$ for the discharge, leading to Equations 3. Though electrical losses are always positive (irreversible heat), the "entropic heat" can be either positive or negative (reversible heat), depending on the signs of the current and the entropy variation.

$$
\begin{gathered}
\dot{Q}_{C h}=I^{2} \times R_{C h}+|I| T_{C h} \frac{\partial U_{o c}}{\partial T}(S o C) \\
\dot{Q}_{D c h}=I^{2} \times R_{D c h}-|I| T_{D c h} \frac{\partial U_{o c}}{\partial T}(S o C)
\end{gathered}
$$

The entropy variation can be computed by subtracting $\dot{Q}_{D c h}$ from $\dot{Q}_{C h}$, leading to Equation (4). In this purpose, they need to be run with the same current rate. Please note that every term is expressed as a function of the SoC.

$$
\frac{\partial U_{o c}}{\partial T}(S o C)=\frac{\dot{Q}_{C h}-\dot{Q}_{D c h}-\overbrace{I^{2} \times\left(R_{C h}-R_{D c h}\right)}^{\Delta \dot{Q}_{\text {Elec }}}}{|I|\left(T_{C h}+T_{D c h}\right)}
$$
by:

From Equation (4), it is thus possible to estimate $\partial U_{o c} / \partial T$

- estimating the heat generations $\dot{Q}_{C h}$ and $\dot{Q}_{D c h}$;

- measuring the temperature $T_{C h}$ and $T_{D c h}$;

- evaluating the difference between electrical losses in charge and discharge (called $\Delta \dot{Q}_{E l e c}$ thereafter).

$\Delta \dot{Q}_{\text {Elec }}$ is not trivial to estimate and thus brings a major source of uncertainty for $\partial U_{o c} / \partial T$ estimation. The precision of the proposed method relies on making $\Delta \dot{Q}_{\text {Elec }}$ as negligible as possible compared to the entropic heat. The entropy variation would hence be approximated by Equation (5).

$$
\frac{\partial U_{o c}}{\partial T}(S o C) \approx \frac{\dot{Q}_{C h}(S o C)-\dot{Q}_{D c h}(S o C)}{|I|\left(T_{C h}(S o C)+T_{D c h}(S o C)\right)}
$$

A first mean to reduce $\Delta \dot{Q}_{\text {Elec }}$ is to increase the temperature of the cell $T$. Thus, the electrical losses decreases, making the entropic heat more significant in the total heat generation. Besides, the entropic heat slightly increases in these conditions.

A second mean to reduce $\Delta \dot{Q}_{E l e c}$ is to decrease the current $I$. As a matter of fact, the entropic heat decreases in proportion to the current and electrical losses in proportion to the square of the current (see Equation (3)). Nevertheless, the cell must generate a minimum of heat to ensure its accurate estimation. Three current rates have been used in the application part and their results will be compared.

The generated heats $\dot{Q}_{C h}$ and $\dot{Q}_{D c h}$ are estimated thanks to a thermal model. The latter and its parameters determinations are presented in the following part.

\section{Heat generation estimation}

\subsection{Thermal model presentation}

A thermal model is usually used for predicting a temperature evolution. It can also be inverted to estimate the generated heat if the external conditions and the temperature evolution are known.

In this study, the cell temperature is assumed to be homogeneous during operation. Hence, it can be measured on its surface. Besides, a one-node thermal model can be 
used (Figure 1). The latter has two parameters: one heat capacity $C_{t h}$ and one thermal resistance $R_{t h}$ toward the cell environment. One equivalent current source represents the heat sources $\dot{Q}$ and an equivalent voltage source is used for the external temperature $T_{\text {ext }}$.

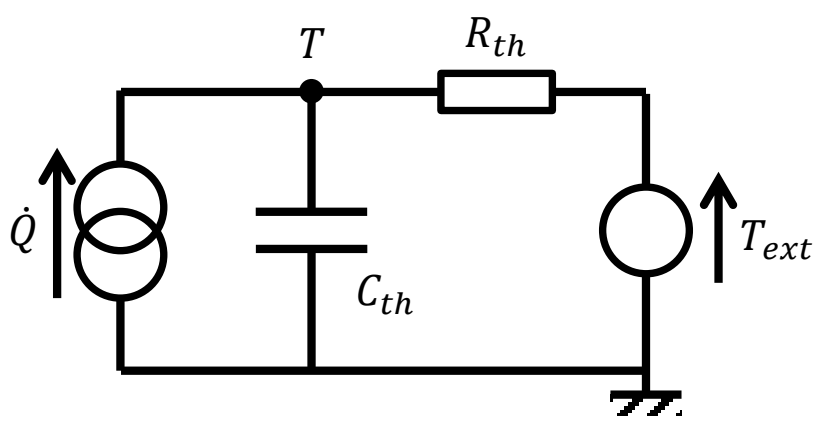

Figure 1: Thermal model of the isothermal cell

The state equation corresponding to this model can be obtained thanks to Kirchhoff's laws (Equation (6)) where $T$ is the temperature of the cell. Before using it to estimate $\dot{Q}_{C h}$ and $\dot{Q}_{D c h}$, the heat capacity $C_{t h}$ and the equivalent thermal resistance $R_{t h}$ values have to be determined.

$$
\dot{Q}=C_{t h} \frac{d T}{d t}+\frac{T-T_{e x t}}{R_{t h}}
$$

The thermal resistance $R_{t h}$ is obtained thanks to the test used for the entropy variation estimation. Its determination will be presented in the section 4 .

\subsection{Heat capacity determination}

As for the heat capacity $C_{t h}$, it has been measured using the setup shown on Figure 2. The cell was connected to a Digatron (BTS-600) that can generate and measure current or voltage with a precision of $0.1 \%$ of the full scale ( $\pm 100 \mathrm{~A}$ with $\pm 20 \mathrm{~V}$ on each of the five circuits). It was packed with insulating materials. As for the upper face, it was covered with glass wool to match the terminals shapes. The five others faces were covered with plates of polyurethane foam. Because power wires are an important cause for thermal leakages [21], they have been wrapped in tubes of rubber foam. A thermocouple has been put at the center of the largest face of the cell (type $\mathrm{T}$, with an absolute precision of $\pm 1{ }^{\circ} \mathrm{C}$ ).

$\mathrm{A} \pm 1 \mathrm{C}$ square current with a period $T$ of $20 \mathrm{~s}$ has been applied to the cell in order to create a heat generation step (a $1 \mathrm{C}$ current fully discharges the battery in $1 h$ ). The 20 -second-long period has been chosen because it is very small compared to the thermal time constant of the cell (being the product of $C_{t h}$ and $R_{t h}$ ). Thus, the average heat generation $\dot{Q}_{a v g}$ can be used in a calculus instead of the instantaneous heat generation. Besides, the $\dot{Q}_{a v g}$ measurement is simple and accurate in these conditions. The SoC and the OCV remain constant and, as the mean current is equal to zero, the mean reversible heat is also equal to zero. Thus, only electrical losses contribute to the

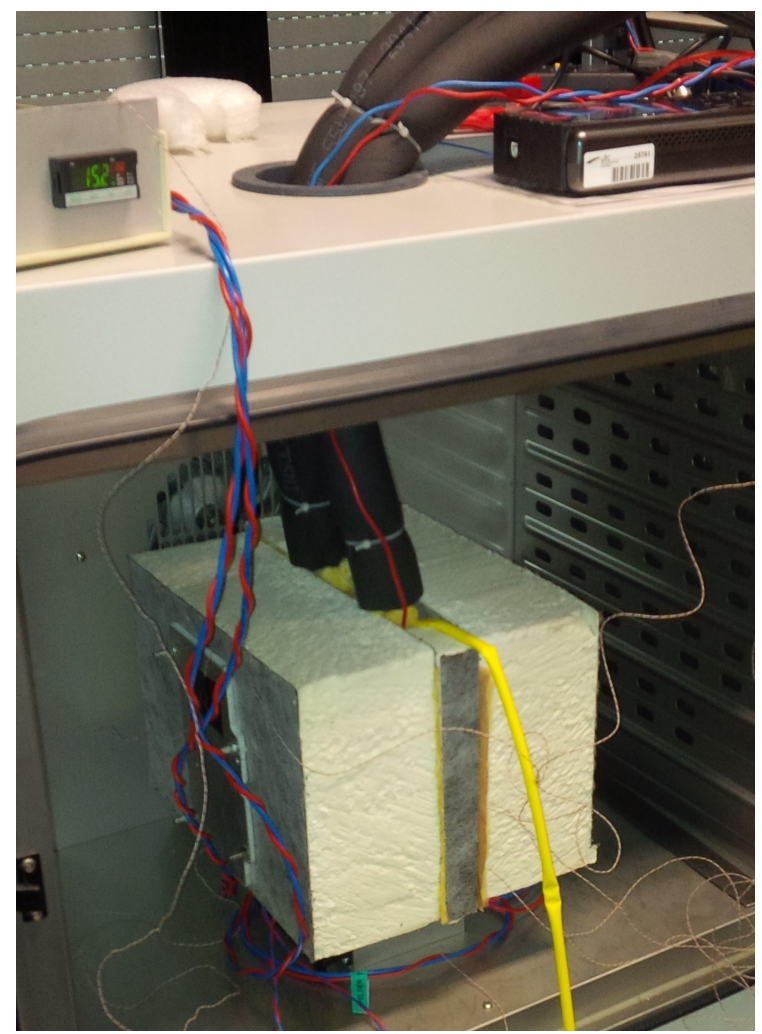

Figure 2: Experimental setup for heat capacity and entropy-variation determination. The cell is covered with insulated material and put within a climate chamber.

cell heating. They can be measured according to Equation (7) by:

- measuring the OCV $U_{o c}$ before beginning the test (the cell being at the equilibrium);

- measuring the current $I_{\text {cell }}$ and the cell voltage $U_{\text {cell }}$ during the test.

$$
\dot{Q}_{\text {avg }}(t)=\frac{1}{T} \int_{t-T / 2}^{t+T / 2} I_{c e l l}\left(U_{c e l l}-U_{o c}\right)
$$

At the beginning of the test, the cell is considered to be in adiabatic conditions and to receive a heat generation step. Consequently, its temperature $T$ increases as a ramp (Figure 3) and the heat capacity can be determined thanks to Equation (8).

$$
C_{t h}=\frac{\dot{Q}_{a v g}}{d T / d t}=1185 J . K^{-1}
$$

\section{Application on a commercial cell}

\subsection{Experimental protocol}

The cell has been insulated as for the heat capacity determination. The aim of this experimental setup is to minimize the cell cooling, so it can be assumed to be isothermal. It has been put in a climatic chamber at $40^{\circ} \mathrm{C}$ and: 


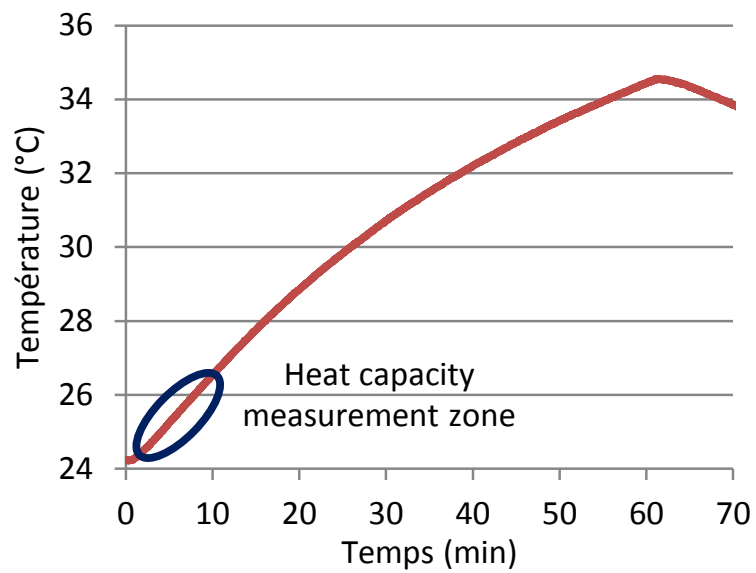

Figure 3: Test for heat capacity determination. Thermal response to a $\pm 1 \mathrm{C}$ square current with a period $T$ of $20 \mathrm{~s}$.

- fully charged by the CCCV method ;

- rested until it reached the thermal equilibrium ;

- fully discharged and rest for several hours ;

- fully charged with the same current and rest for several hours.

Between each step, the cell has been kept in open circuit until its temperature reaches the chamber temperature $\left( \pm 1^{\circ} \mathrm{C}\right)$. This keeps it from overheating and it allows the accurate determination of $R_{t h}$.

\subsection{Thermal resistance determination}

The temperature evolution of the cell and the corresponding heat generation have been reported on Figure 4 for the $0.75 \mathrm{C}$ test. Whereas the climatic chamber temperature had been set to $40^{\circ} \mathrm{C}$, the equilibrium temperature of the cell was $37^{\circ} \mathrm{C}$. This is due to the power wires, which create a thermal link with the ambient temperature of the test room (being $20^{\circ} \mathrm{C}$ ). In order to keep the thermal model and its parameter determination as simple as possible, the external temperature $T_{\text {ext }}$ in Equation (6) is replaced by the equilibrium temperature $T_{e q}$ (Equation (9)).

$$
\dot{Q}=C_{t h} \frac{d T}{d t}+\frac{T-T_{e q}}{R_{t h}}
$$

By doing so, the model still has only one equivalent thermal resistance $R_{t h}$. Its value has been found to be $7.67 K . W^{-1}$ thanks to an optimization algorithm run during the first cooling phase.

\subsection{Heat generation estimation}

All the parameters of the thermal model being determined, the heat generation reproduced on Figure 4 can be estimated through Equation (9). $T$ and $T_{e q}$ were measured and the derivative $d T / d t$ was estimated by a linear regression, carried on a time interval of $300 \mathrm{~s}$ centered on the calculation points.

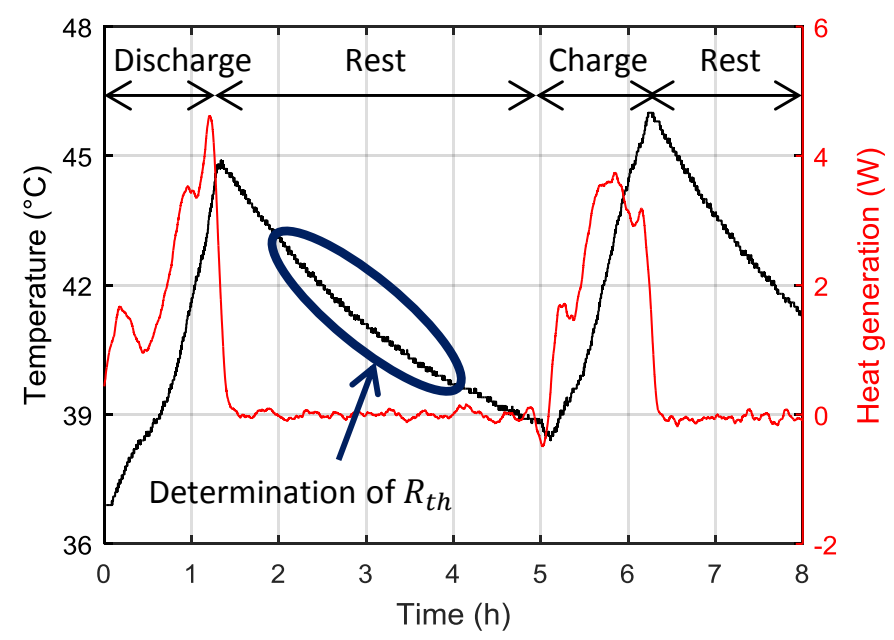

Figure 4: Thermal response and measured heat generation for $0.75 \mathrm{C}$ dicharge and charge.

A "noise" on the heat generation estimation can be observed during the rest phases. It is relatively low compared with the generated heat (about $0.1 W$ ) thanks to the long time range used for estimating the derivative of $T$. However, the quasi-instantaneous heat-generation drops at the charge and discharge ends are smoothed.

\subsection{Results and discussions}

During the charge and the discharge, large variations of the heat generation can be observed (from $-0.5 \mathrm{~W}$ to $5 W)$. These variations are mostly caused by the entropic heat. This has been highlighted by plotting the heats estimated thanks to Equation (9) in charge (orange square marks) and discharge (red circle marks) as functions of the SoC (Figure 5). By doing so, both electrical losses (green downward-pointing triangle marks) and entropic heat (blue upward-pointing triangle marks) appear (see Equations 3).

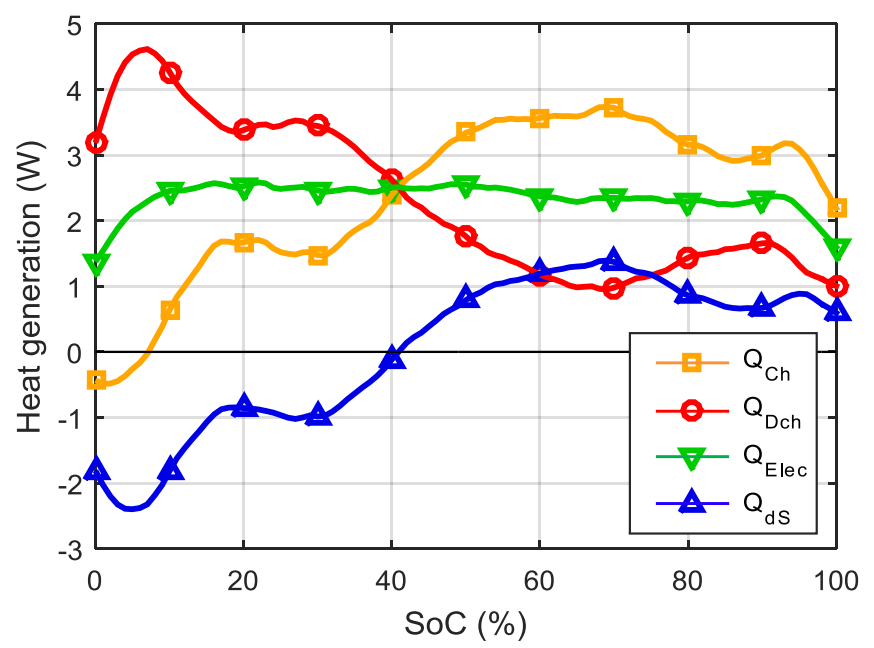

Figure 5: Heat generation in charge and discharge, entropic heat and electrical losses ( $0.75 \mathrm{C}$ test). 
The estimated heat generations are consistent, except below $5 \%$ SoC and above $95 \%$ SoC. They are biased in these $\mathrm{SoC}$ ranges, because the $T$ derivative calculus smooths their quick variations. Between $5 \%$ and $95 \%$, the computed electrical losses are nearly constant (about $2.5 \mathrm{~W})$ and the extracted entropic heat $\dot{Q}_{d S}$ shape is typical of a $\mathrm{LiFePO}_{4} /$ graphite cell $[24,25]$. The corresponding entropy variation has been reported on Figure 6 , along with the results obtained via $\mathrm{C} / 2$ and $1 \mathrm{C}$ tests. Some points of $\partial U_{o c} / \partial T$ have been measured using the potentiometric method and added to the Figure 6 .

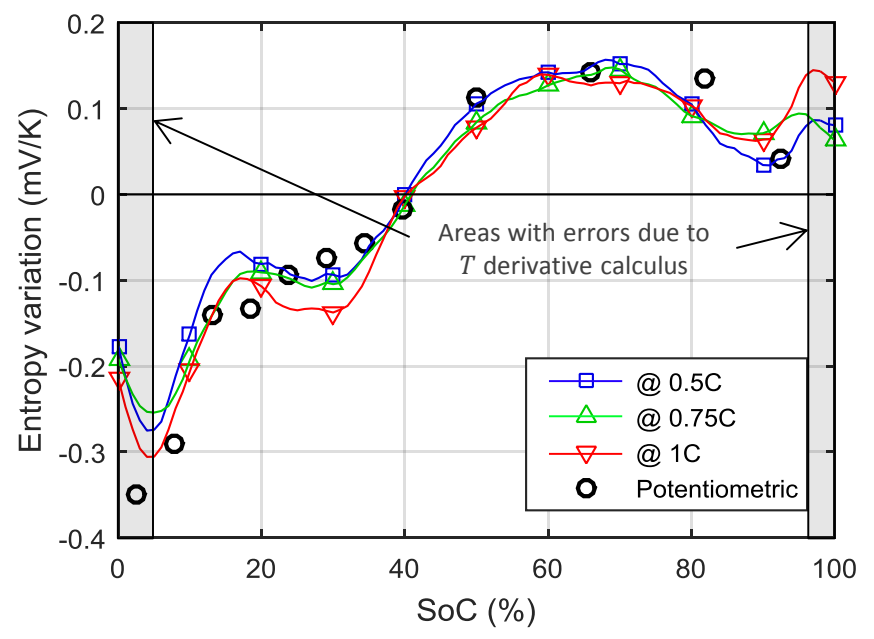

Figure 6: Entropy variation of a commercial LiFePO4/graphite cell, obtained with the proposed method for three different current rates and obtained using the classical potentiometric method.

The three current rates lead to close entropy-variation curves. Moreover, the latters are consistent with the values obtained thanks to the potentiometric method. This confirms that neglecting $\Delta \dot{Q}_{E l e c}$ is reasonable. In this view, results obtained through $\mathrm{C} / 2$ charge and discharge are bound to be the most precise because the electrical losses are at their lowest compared to entropic heat.

\section{Conclusion}

The proposed calorimetric method allows a fast and precise determination of a battery entropy-variation as function of its state of charge. The experiment needed only takes a few hours and the corresponding low-cost experimental setup can also be used for the heat capacity measurement (the latter being needed in the experimental protocol). A minimal knowledge is required on the cell to be characterized: there is no need to determine neither its OCV curve nor its electrical properties. Thus, this method is well adapted for industries and laboratories. Moreover, the results are nearly continuous where the classical potentiometric method can only give discrete values after several days or week of tests.

This work has been validated with an $\mathrm{LiFePO}_{4} /$ graphite battery, but it is also usable for aged batteries or for any other technology as long as electrical losses and entropic heat are the main heat sources and if the difference between electrical losses in charge and discharge can be neglected. This second hypothesis may be verified by comparing results obtained with different current rates.

The heat generation estimation obtained by inverting a simple thermal model gives good results on an isolated cell hence avoiding the use of a calorimeter. It could be further improved by using a temperature sensor having a better resolution. In this paper, the $0.1{ }^{\circ} \mathrm{C}$ resolution of the sensor implied the use of a large time interval for the temperature derivative estimation (to reject the noise due to the "stair effect"). A better resolution of the sensor would allow a narrower time interval for the derivative calculus and, consequently, it would bring more detailed entropy-variations curves.

\section{Acknowledgement}

The authors acknowledge the ANRT for its financial support, the company E4V for its participation to the study and Yves Chabre for his scientific support.

\section{References}

[1] E. Prada, Aging modeling and lifetime optimization of Li-ion $\mathrm{LiFePO}_{4}$-graphite batteries according to the vehicle use, Ph.D. thesis (2013).

[2] M. T. Lawder, P. W. C. Northrop, V. R. Subramanian, ModelBased SEI Layer Growth and Capacity Fade Analysis for EV and PHEV Batteries and Drive Cycles, Journal of the Electrochemical Society 161 (14) (2014) A2099-A2108.

[3] C. Edouard, M. Petit, J. Bernard, C. Forgez, R. Revel, Sensitivity Analysis of an Electrochemical Model of Li-ion Batteries and Consequences on the Modeled Aging Mechanisms, ECS Transactions 66 (9) (2015) 37-46.

[4] A. Cordoba-Arenas, S. Onori, G. Rizzoni, A control-oriented lithium-ion battery pack model for plug-in hybrid electric vehicle cycle-life studies and system design with consideration of health management, Journal of Power Sources 279 (2015) 791808.

[5] T. R. Tanim, C. D. Rahn, Aging formula for lithium ion batteries with solid electrolyte interphase layer growth, Journal of Power Sources 294 (2015) 239-247.

[6] C. Alaoui, Solid-State Thermal Management for Lithium-Ion EV Batteries, IEEE Transactions on Vehicular Technology 62 (1) (2013) 98-107.

[7] S. Al Hallaj, H. Maleki, J.-S. Hong, J. R. Selman, Thermal modeling and design considerations of lithium-ion batteries, Journal of Power Sources 83 (1999) 1-8.

[8] C. Forgez, D. Vinh Do, G. Friedrich, M. Morcrette, C. Delacourt, Thermal modeling of a cylindrical $\mathrm{LiFePO}_{4} /$ graphite lithium-ion battery, Journal of Power Sources 195 (9) (2010) 2961-2968.

[9] X. Lin, H. E. Perez, S. Mohan, J. B. Siegel, A. G. Stefanopoulou, Y. Ding, M. P. Castanier, A lumped-parameter electro-thermal model for cylindrical batteries, Journal of Power Sources 257 (2014) $1-11$.

[10] C. Lin, K. Chen, F. Sun, Research on thermo-physical properties identification and thermal analysis of EV Li-ion battery, Vehicle Power and Propulsion Conference (VPPC), IEEE (2009) 1643-1648.

[11] A. Pruteanu, B. V. Florean, G. M. Moraru, R. C. Ciobanu, Development of a thermal simulation and testing model for a 
superior lithium-ion-polymer battery, Optimization of Electrical and Electronic Equipment (OPTIM), IEEE (2012) 947-952.

[12] K. Murashko, J. Pyrhonen, L. Laurila, Three-Dimensional Thermal Model of a Lithium Ion Battery for Hybrid Mobile Working Machines: Determination of the Model Parameters in a Pouch Cell, IEEE Transactions on Energy Conversion 28 (2) (2013) 335-343.

[13] K\&K Associates, Thermal Network Modeling Handbook, K\&K Associates, Westminster, 1999.

[14] N. Watrin, H. Ostermann, B. Blunier, A. Miraoui, Multiphysical Lithium-Based Battery Model for Use in State-of-Charge Determination, IEEE Transactions on Vehicular Technology 61 (8) (2012) 3420-3429.

[15] M. Fleckenstein, S. Fischer, O. Bohlen, B. Bäker, Thermal Impedance Spectroscopy - A method for the thermal characterization of high power battery cells, Journal of Power Sources 223 (2013) 259-267.

[16] K. Chen, G. Unsworth, X. Li, Measurements of heat generation in prismatic Li-ion batteries, Journal of Power Sources 261 (2014) 28-37.

[17] P. Amiribavandpour, W. Shen, D. Mu, A. Kapoor, An improved theoretical electrochemical-thermal modelling of lithium-ion battery packs in electric vehicles, Journal of Power Sources 284 (2015) 328-338.

[18] K. E. Thomas, J. Newman, Heats of mixing and of entropy in porous insertion electrodes, Journal of Power Sources 119-121 (2003) 844-849.

[19] L. Gagneur, A. Driemeyer-Franco, C. Forgez, G. Friedrich, Modeling of the diffusion phenomenon in a lithium-ion cell using frequency or time domain identification, Microelectronics Reliability 53 (6) (2013) 784-796.

[20] J. Illig, Physically based Impedance Modelling of Lithium-ion Cells, Ph.D. thesis (2014).

[21] N. Damay, C. Forgez, M.-P. Bichat, G. Friedrich, Thermal modeling of large prismatic $\mathrm{LiFePO}_{4} /$ graphite battery . Coupled thermal and heat generation models for characterization and simulation, Journal of Power Sources 283 (2015) 37-45.

[22] Y. F. Reynier, R. Yazami, B. Fultz, Thermodynamics of Lithium Intercalation into Graphites and Disordered Carbons, Journal of The Electrochemical Society 151 (3) (2004) A422A426.

[23] R. E. Williford, V. V. Viswanathan, J.-G. Zhang, Effects of entropy changes in anodes and cathodes on the thermal behavior of lithium ion batteries, Journal of Power Sources 189 (1) (2009) 101-107.

[24] V. V. Viswanathan, D. Choi, D. Wang, W. Xu, S. Towne, R. E. Williford, J.-G. Zhang, J. Liu, Z. Yang, Effect of entropy change of lithium intercalation in cathodes and anodes on Li-ion battery thermal management, Journal of Power Sources 195 (11) (2010) $3720-3729$.

[25] J. P. Schmidt, A. Weber, E. Ivers-Tiffée, A novel and precise measuring method for the entropy of lithium-ion cells: $\Delta S$ via electrothermal impedance spectroscopy, Electrochimica Acta 137 (2014) 311-319. 